\title{
Un modelo de vivienda tradicional en el San Fernando del siglo XVIII
}

\section{JOSÉ M. ${ }^{a}$ MOLINA MARTÍNEZ Universidad de Cádiz}

Es conocido que el actual San Fernando sólo era a fines del s. XVII, como apunta Salvador Clavijo, "Una seriación dispersa de edificios y caseríos, 'con raigambre bien calificable» (Clavijo', S., 1961). Hasta entonces, las quintas de recreo de los gaditanos adinerados salpicaban esporádicamente el territorio isleño, y sólo el camino que unía el Castillo de León (hoy denominado de San Romualdo) con la iglesia del Carmen ponía cierto orden en tan anárquica distribución de edificios.

La entrada del nuevo siglo supondrá, tanto para la Isla como para el resto de poblaciones ribereñas a la Bahía, un cambio sustancial en las estructuras demográficas, económicas y sociales mantenidas hasta entonces; ello conllevará, en el caso particular de San Fernando, un incremento de su nivel poblacional, derivado de una importante inmigración y de un fuerte aumento de la natalidad (1). Este crecimiento de la población determina, como es lógico, un aumento de las construcciones, que básicamente responderán a dos características: su poca consistencia y su ubicación en torno a las edificaciones más significadas del término isleño.

Como resultado de todo el proceso aparecen las primeras agrupaciones de casas o conatos de barrios que iran consolidándose como tales a medida que vaya transcurriendo el siglo y aumentandó el número de habitantes de la Isla.

La fuente documental básica con la que contamos para la elaboración de nuestro trabajo son las series parroquiales contenidas en el $\mathrm{Ar}-$ chivo de la iglesia Mayor de San Fernando (2). De estas series, y en concreto de las correspondientes a los libros de defunciones, extraemos una serie de datos (domicilio del fallecido, situación geográfica de la casa que

(1) Trabajo en claboración.

(2) Archivo Iglesia Mayor Parroquial, libros de defunciones números, 1, 2, 3, 4 y 5. 
habitaba, etc.) que nos aclararán la ubicación de los primeros barrios isleños.

Clavijo y Clavijo nos detalla en su obra cuál era la situación urbana de la Isla a fines del s. XVII (3). Sin embargo, en su análisis faltan alusiones a estas primeras agrupaciones de casas o "barrios", que si constan con tal denominación en las fuentes parroquiales, en concreto aparecen como tales dos: el denominado de "Vidal" y el de la "Huerta Perdida". En uno de estos barrios, "el de Vidal", centramos nuestro estudio, ya que en la zona que ocupaba en el s. XVIII aún queda un ejemplo del tipo de vivienda que intentamos analizar.

En el plano de Fray Jerónimo de la Concepción (Concepción, F. J. de la, 1690) aparece con este nombre (Vidal) una extensión de terreno situada en el cuadrante noroccidental del término isleño. Era su propietario don Pedro Vidal y Saavedra, un vecino rico de la ciudad Cádiz (Cristelln, J., 1891) que a fines del s. XVII sólo debía poseer los terrenos, ya que los planos de la época no reflejan ningún tipo de construcción en su propiedad (4); además, en las actas de defunciones de las personas que vivían en aquel lugar nunca se hace referencia al tipo de vivienda que habitaba, información esta, que dándose normalmente en todas las partidas se soluciona en estos casos con un escueto "vivía en el manchón propiedad de Don Pedro Vidal, vecino de Cádiz» (5). Sin embargo, en las partidas de fines del s. XVII y principios del s. XVIII, ya aparecen alusiones a las residencias de estas personas fallecidas; así, entre los años 1696 y 1703 la documentación nos expresa ya tipos muy concretos de vivienda como el "Corralón", "el patio de vecindad" o "las accesorias", siendo este último tipo de habitat el más repetido entre las personas que habitaban el lugar. Es evidente, por tanto, que en la zona o manchón de Vidal se construyeron las primeras casas a partir del último lustro del s. XVII.

La documentación, interrumpida entre los años 1708 y 1715 nos impide seguir la evolución de la zona durante estos primeros años del s. XVIII. Evolución que sin duda debió ser importante, ya que en el mismo año que se recupera la serie (1715) son abundantes las actas que hacen referencia al lugar, denominándosele ya como «barrio de Vidal» (6).

(3) El estudio lo realiza Clavijo en base a los datos suministrados por el plano de F.J. de la Concepción y por el mapa original de la Isla de León de 1660 (levantado en 1852) existente en el archivo de la familia Lobo.

(4) Nos referimos al plano de Fray Jerónimo de la Concepción y al levantado por el almirante Lobo.

(5) Archivo Iglesia Mayor Parroquial, defunciones, libro 2. ${ }^{\circ}$, p. 29 v.

(6) Archivo Iglesia Mayor Parroquial, defunciones, libro $3 .^{\circ}$. 
Sin lugar a dudas, la proyección de la zona es evidente: en veinticinco años, quizás menos, ha pasado de terreno para pastos a un lugar ocupado, que ya merece el título de barrio y que según hemos podido comprobar por la documentación parroquial está compuesto fundamentalmente por unos tipos de viviendas (corralones, patios de vecindad, accesorias...) poco propias de un medio rural como era la Isla de León a principios del s. XVIII. Una de estas viviendas, situada en el extremo norte de la calle González Hontoria (7) y que en la actualidad se encuentra en un lamentable estado (sólo mantiene en pie sus muros exteriores) es la que hemos elegido para intentar llevar a cabo nuestro estudio.

Sin embargo, hemos de advertir que la distribución de la vivienda efectuada en base a los escasos indicios ofrecidos por los elementos materiales que aún quedan en pie, debe entrar a veces en el campo de la especulación. No obstante, y a pesar de que somos conscientes del peligro que ello entraña, pretendemos aportar algunos datos al enorme vacío existente en este campo.

La vivienda está ubicada en el cruce de cuatro calles: San Esteban, Constructora Naval, González Hontoria y Antonio López, de la que forma parte una de sus fachadas, mientras la segunda se orienta a una pequeña plaza formada en la intersección de estas calles (8). Parece haber sido edificada en varios momentos, ya que ni sus puertas ni ventanas exteriores, ni sus muros, guardan lógica alguna si tomamos el edificio como todo un conjunto. En cambio, si diferenciamos la casa en varios módulos, estos elementos sí aparecerían coherentemente dispuestos.

Un segundo elemento a tener en cuenta es la información que recogen las fuentes parroquiales sobre los tipos de vivienda incluidos en el barrio. En nuestro caso, las reducidas dimensiones del edificio, y la disposición de los restos de muros, dejan en el interior unos espacios abiertos o posibles patios excesivamente pequeños como para pensar que fuese cualquiera de los tipos de corralón o patio de vecindad. En cuanto a la Casería, la vivienda en su conjunto sí ofrece similitud con un edificio de campo; sin embargo, la gran cantidad de puertas que se abren al exterior y el hecho de que en la documentación se mencione en un momento cronológico más tardío que el resto de las viviendas, hacen inviable esa posibilidad, por lo que sólo nos quedaría la casa de accesorias como único tipo de vivienda donde encajar los restos en estudio.

(7) Esta calle, oficialmente rotulada como González Hontoria, es conocida popularmente como calle Vidal, lo que confirma la situación de la casa que analizamos en el anteriormente denominado "barrios de Vidal».

(8) Ver plano adjunto. 
Así pues, y partiendo de estas premisas, nos aproximaremos a lo que podría ser su aspecto exterior y su distribución interior hace casi tres siglos.

El edificio que hemos identificado como casa de accesorias, es decir, una casa que sin llegar a ser patio de vecindad dispone de pequeños habitáculos de no más de tres habitaciones, donde una familia vivía independizada del resto de la comunidad que habitaba la totalidad de la vivienda, parece, como ya hemos afirmado, haber sido construida en tres períodos distintos; de hecho, y aparte de las características antes expresadas, la fachada ofrece salientes y entrantes en las dos caras del edificio.

Así pues, y analizando la planta de distribución general (9) y la planta de cubierta (10), observamos que tanto la fachada norte como la este ofrecen salientes que diferencian en tres pequeños módulos el conjunto de la casa.

El primero de ellos (A), formado por tres habitaciones que en total supone unos veinte metros cuadrados, dispondría de dos huecos a la calle: una puerta, actualmente tapiada, y una ventana de aproximadamente $2,5+1,5$ metros resguardada por una reja de hierro. Hacia el interior poseía tres huecos abiertos a un supuesto patio. Las tres habitaciones que componen esta vivienda estaban comunicadas entre sí, y todas ellas cubiertas por una azotea rematada en las esquinas de sus pretiles por sencillos elementos decorativos, remates que, junto a la estructura de la fachada, nos hicieron identificar el módulo como el último en añadirse al conjunto del edificio.

El segundo de estos módulos (8) dispone de dos fachadas, pues en esta parte es donde el edificio hace esquina. En su conjunto esta parte de la casa estaría compuesta por tres viviendas paralelas unas a otras, formada cada una de ellas de dos habitaciones, una abierta a la calle a través de la puerta de entrada (actualmente cerrada), y la segunda, que por la forma de los restos de muros suponemos tendría algún hueco al patio interior. En el caso de la vivienda más cercana a la esquina del edificio, el hueco del patio se abre en la fachada norte, ya que el final de la segunda habitación es muro frontero con el del módulo antes analizado. En cuanto a cubiertas, sólo una de las tres viviendas que componen el módulo parece haber sido rematada con cubierta de tejas de un solo pendiente hacia la fachada norte, recogiéndose el agua en un canalón que la

(9) Apéndice plano 3 .

(10) Apéndice plano 4 
transportaba a la calle, quedando el resto cerradas por azoteas sin remates en los pretiles.

Toda esta parte del conjunto, debido a las reducidas dimensiones de sus habitaciones, a la disposición en esquina de sus fachadas y a los elementos exteriores: un banco de piedra a lo largo de la fachada norte y dos escalones que preceden a cada una de las puertas de entrada a las viviendas en la fachada este, parecen indicar que fue éste, el primer módulo èn construirse; módulo al que se le añadió posteriormente y continuando cada una de sus fachadas el resto de viviendas que completa el conjunto.

Por último, la tercera de estas partes (C) estaría compuesta de dos viviendas, dispuestas como el caso anterior en paralelo, aunque separadas por un pequeño patio y con habitaciones más amplias que las anteriores; la primera de ellas colindante al módulo anterior, dispondría de dos habitaciones muy ventiladas: la exterior por la puerta de salida y una ventana al patio divisorio, y la segunda por un hueco trasero al patio general y otro al divisorio. La segunda vivienda estaría compuesta de tres habitaciones, una de ellas superpuesta en un piso elevado sobre la habitación de entrada, dispone de ventilación a la calle a través de una pequeña ventana. En la planta baja, la vivienda se distribuye de forma simétrica a la anterior, teniendo los mismos huecos interiores que aquella; es decir, dos al patio divisorio y uno al general. Las cubiertas de esta parte son de tejas con caída en una sola pendiente. En definitiva, el conjunto de este tercer módulo, que creemos es el primer añadido al edificio original (8), es en su conjunto de mayor envergadura que los analizados anteriormente.

En cuanto a materiales, el edificio, que globalmente tiene forma rectangular y ocupa un espacio de aproximadamente unos doscientos cincuenta metros cuadrados, parece estar cimentado con una zapata corrida a lo largo de todo el perímetro, compuesta probablemente y por el período y lugar geográfico que estudiamos, por cascotes de roca ostione$\mathrm{ra}$, formando masa con un aglomerante y tierra zahorra; elementos, que junto al ladrillo-taco de adobe conforman la cimentación de una zapata interior dispuesta bajo los teóricos muros de carga, que irían revestidos exteriormente con una mezcla de cal y arena para finalmente ser enlucidos con cal.

En lo que respecta a suelos y techos, en el primero de los casos las habitaciones llevarían una solería de algún material cerámico de baja calidad, y en el segundo están compuestos al interior por vigas de madera apoyadas en los muros laterales, cubriéndose exteriormente con baldosas o ladrillos cerámicos de gran poder absorvente. En las partes de la 
vivienda que se coronan con cubiertas de tejas, éstas van apoyadas en viguería de madera.

En resumen, se trata de un tipo de vivienda edificada sin grandes pretensiones y cuya finalidad es dar cabida al mayor número de familias en el menor espacio posible, con el fin de solucionar el doble problema que afecta al lugar a principios del s. XVIII: por un lado, dar cobijo a la fuerte inmigración que registra la zona en estas fechas, y por otro, acomodarse a las pretensiones capitalinas que desde un siglo atrás prohibía hacer nuevas edificaciones en la Isla (Corzo, R., 1980).

\section{FUENTES}

Archivo Iglesia Mayor de San Pedro y San Pablo. San Fernando.

Defunciones, libros I (1680-1701) y II (1701-1729).

Matrimonios, libros I (1680-1692), II (1692-1713) y IV (1724-1754).

Bautizos, libros I y II (1656-1697).

\section{BIBLIOGRAFÍA CITADA}

CLAVIJO, S. (1961), La ciudad de San Fernando, Historia y Espíitu. San Fernando, $590 \mathrm{pp}$.

CONCEPCIÓN, F.J. de la, (1690), Emporio del Orbe, Cádiz Ilustrada. Amsterdam. 700 pp.

CORZO, R., (1980), San Fernando. Col. Historia de los pueblos de la provincia de Cádiz. Cádiz. $131 \mathrm{pp}$.

CRISTELLY, J. (1891). Ligeros apuntes históricos de la ciudad de San Fernando desde los tiempos más remotos hasta el año 1823. San Fernando. 200 pp. 